Journal of Animal and Veterinary Advances 11 (19): 3535-3538, 2012

ISSN: $1680-5593$

(C) Medwell Journals, 2012

\title{
Measurement of Keratan Sulfate in Plasma from Sow and Piglets
}

\author{
${ }^{1}$ Sachiko Arai, ${ }^{3}$ Keiji Takahashi, ${ }^{2}$ Fujiko Sunaga and ${ }^{1}$ Seigo Itoh \\ ${ }^{1}$ Department of Internal Medicine, ${ }^{2}$ Department of Infectious Disease, \\ School of Veterinary Medicine, Azabu University, \\ 1-17-71 Fuchinobe, Chuo-ku, Sagamihara-shi, 252-5201 Kanagawa, 042-75, Japan \\ ${ }^{3}$ Chiba Prefectural Livestock Reserach Center, Livestock Production Technology Division, \\ Swine and Poultry Laboratory, 16-1, He, Yachimata, Yachimata-shi, 289-113 Chiba, Japan
}

\begin{abstract}
Leg weakness is a regressive locomotory disease associated with osteochondrosis and osteoarthritis. However, there are no marked clinical symptoms and it is difficult to evaluate the prognosis or make a diagnosis before death. In humans joint markers to measure cartilage substances in blood are utilized. In particular, keratan sulfate specifically exists in cartilage. This parameter has been measured in dogs and horses. However, no study has reported measurement in pigs. Furthermore, age-/breed-related or gender differences are unclear. In this study, reserachers clarified the hemodynamics of keratan sulfate in pigs and confirmed that the keratan sulfate level decreased when leg weakness was clinically observed in the sow and growing pigs for breeding. In sows the blood level of keratan sulfate decreased to about $1 / 10$ of that in piglets. This was possibly because a pig-specific breeding form with the stall was employed in sows affecting articular movement and reducing cartilage metabolism.
\end{abstract}

$\underline{\text { Key words: Keratan sulfate, pigs, locomotory disease, osteochondrosis, osteoarthritis }}$

\section{INTRODUCTION}

Leg weakness in pigs refers to non-infectious arthritis related to inheritance rapid growth/weight gain or physical stimulation of joints. Primary etiological factors include osteochondrosis and osteoarthritis (Reiland, 1978; Furugouri, 1984; Kusuhara, 1999). These disorders which cause leg weakness are localized in the articular cartilage. Osteochondrosis and osteoarthritis are associated with micronecrosis of the articular cartilage. However, persistent imbalanced cartilage loading or excessive forces added to joints lead to the enlargement of osteochondrosis lesions making osteoarthritis lesions more serious. In an experimental pig leg weakness model which we reported clinical symptoms such as claudication and pain serially subsided. However, cartilage lesions may progressively deteriorate (Arai et al., 2009, 2010). Therefore, it is necessary to detect leg weakness in the early stage. However, in most cases these lesions are detected after death or slaughter. In humans techniques to measure/quantify articular cartilage components and enzymes involved in cartilage destruction using patient blood or synovial fluid have been developed to clarify the pathogeneses of articular diseases such as arthritis and osteoarthritis. Some of these joint markers have been utilized in clinical practice (Levick, 1990; Kikuchi and Hayakawa, 1992). In the presence of articular diseases, Proteoglycan (PG) contained in the articular cartilage is decomposed in the process of articular destruction. A decomposition-related product Glycosaminoglycan (GAG) diffuses into body fluid such as synovial fluid, blood and urine. In particular a type of GAG keratan sulfate is contained in the cornea other than the articular cartilage at only a small quantity in vivo. This component specifically reflects the degree of cartilage metabolism and articular destruction. For this reason, the synovial fluid and blood levels of keratan sulfate are measured as indices for the diagnosis and treatment monitoring of articular diseases such as Osteoarthritis (OA) and Rheumatoid Arthritis (RA) in humans (Hanashima et al., 1997). Keratan sulfate can be measured using patient serum, plasma and synovial fluid. ELISA kits for humans are commercially available, facilitating simple measurement. Therefore, initially, researchers examined whether the pig plasma level of keratan sulfate can be measured using a commercially available ELISA kit for humans. In addition, there were marked changes in the blood level of keratan sulfate at a young age in humans (Thonar et al., 1988) dogs (Fujiki et al., 2006) and horses (Okumura et al., 2002). This suggests that age and

Corresponding Author: Seigo Itoh, Department of Internal medicine, School of Veterinary Medicine, Azabu University, 1-17-71 Fuchinobe, Chuo-ku, Sagamihara-shi, 252-5201 Kanagawa, 042-75, Japan 
previous delivery must be considered for the clinical diagnosis of leg weakness in pigs. In this experiment researchers compared the keratan sulfate level between adult pigs and piglets clarified its changes in piglets and evaluated its changes in pigs with the spontaneous onset of limb disturbance.

\section{MATERIALS AND METHODS}

Twelve Landrace $\mathrm{x}$ Large White breeder pigs (including 9 gilts) raised at Chiba Prefectural Livestock Research Center and Azabu University were used. Five of the 12 pigs were healthy in appearance with no abnormality of the legs or hoofs whereas 7 pigs manifested abnormalities in the hoofs and gait and were clinically diagnosed with leg weakness. Blood samples were collected from the jugular vein and immediately centrifuged and serum was collected.

Serum was 20 fold diluted and subjected to keratan sulfate measurement using an ELISA kit for humans (Nippon Chemical Industrial Co., Ltd.). One young pig with hoof disorder showed a poor condition due to an unfavorable gait on the landing of the right front and left hind legs. This animal was sacrificed and autopsied and the distal femoral epiphysis was macroscopically observed employing the India Ink Method and compared with the serum keratan sulfate level.

In 26 Landrace piglets produced/reared as breeding pig candidates at the Chiba Prefectural Livestock Research Center, blood was regularly collected between 39 and 164 days of age. Their parent pigs were produced through a combining ability test with 3 types of Landrace boar with strong healthy limbs.

\section{RESULTS AND DISCUSSION}

Results of comparison of the keratan sulfate level by parity or leg condition shows were Table 1 . The mean keratan sulfate levels in animals with and without leg weakness were $22.3 \pm 7.5$ and $29.5 \pm 10.7 \mathrm{ng} \mathrm{mL}^{-1}$, respectively. When the pigs were divided into young (gilts, $n=8$ ) and elderly ( 5 or more parity's, $n=4$ ) animals, the mean keratan sulfate levels were $28.8 \pm 9.6$ and $18.4 \pm 2.6 \mathrm{ng} \mathrm{mL} \mathrm{mL}^{-1}$, respectively. In the young pigs, the mean keratan sulfate levels in animals with and without leg weakness were $24.3 \pm 7.9(\mathrm{n}=5)$ and $35.2 \pm 8.0 \mathrm{ng} \mathrm{mL}^{-1}$ $(\mathrm{n}=3)$, respectively. In the elderly pigs, the mean keratan sulfate levels in animals with and without leg weakness were $17.3 \pm 3.8(n=2)$ and $19.6 \pm 1.3 \mathrm{ng} \mathrm{mL}-1(n=2)$, respectively.

Result of comparison of the keratan sulfate level and autopsy findings in a pig with leg weakness (Fig. 1 and 2). The sacrificed pig was autopsied. The sole of the

\begin{tabular}{|c|c|c|c|}
\hline \multirow[b]{2}{*}{ Conditions } & \multicolumn{3}{|c|}{ Keratan sulfate concentration (ng $\mathrm{mL}^{-1}$ ) } \\
\hline & Gilt (n) & Sow (n) & Total (n) \\
\hline Normal & $35.2 \pm 8.0$ & $19.6 \pm 1.3(2)$ & $29.5 \pm 10.79(5)$ \\
\hline Legweakness & $24.3 \pm 7.9(5)$ & $17.3 \pm 3.8(2)$ & $22.3 \pm 7.5(7)$ \\
\hline Total & $28.8 \pm 9.6(8)$ & $18.4 \pm 2.6(4)$ & - \\
\hline
\end{tabular}

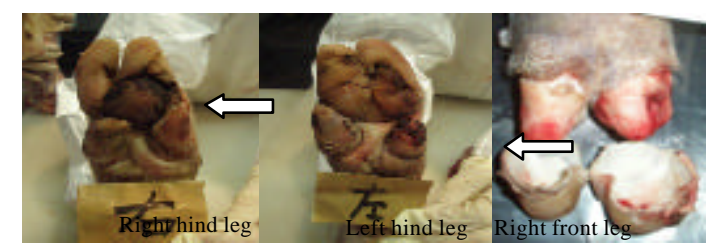

Fig. 1: Autopsy of the legs of a pig sacrificed due to hoof disorders. Right hind leg: wide erosion and deep cracks were noted in the right hind sole (White arrow). Left hind leg: the declaw was defective in the left hind leg (white arrow). Right front leg: deep ulcerative lesions were present in the right frontal hoof wall and sole (circled)

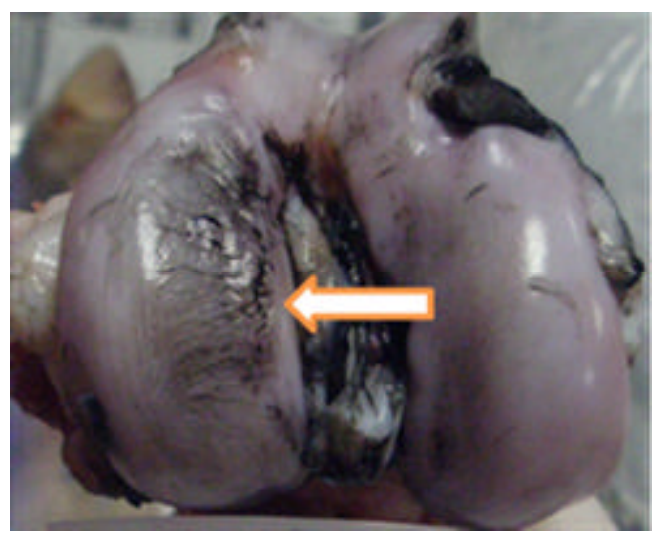

Fig. 2: The distal femoral epiphysis of the left hind leg in a gilt sacrificed due to hoof disorder (India Ink Method). Crack-like lesions were present on the medial side of the joint (arrow). This is a macroscopic feature of osteoarthropathy

right front hoof had eroded widely and deep ulcerative lesions reaching the dermis were present in the hoof wall and sole. Cracks were present in the cartilage surface of the distal femoral epiphysis of the left hind leg which is a macroscopic feature of osteoarthritis. The serum keratan sulfate level was $23.3 \mathrm{ng} \mathrm{mL} \mathrm{m}^{-1}$ in this pig, being lower than the mean level in the young pigs.

Changes in the keratan sulfate level in plasma from piglets: The serial changes in the plasma level of keratan sulfate in piglets as well as the serial changes in the cartilage thickness (Nakano et al., 1979) are shown in 


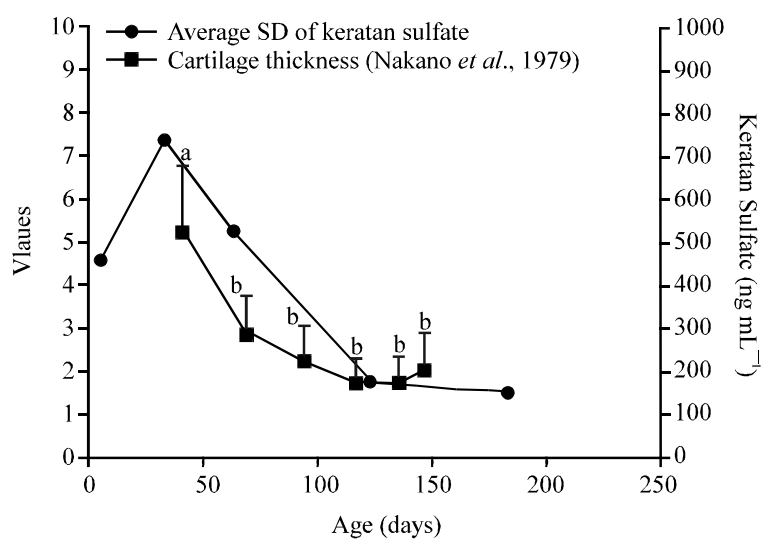

Fig. 3: Serial changes in the plasma KS level and cartilage thickness

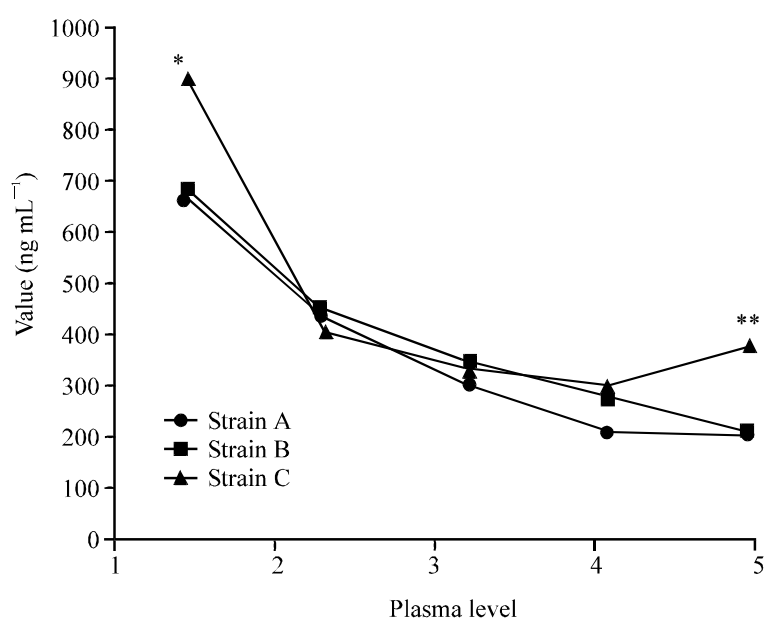

Fig. 4: Comparison of the plasma level of keratan sulfate among stains. ${ }^{* *}$ Strain C vs. A, B: $\mathrm{p}<0.05$; $* *$ Strain C vs. A, B: $p<0.01$

Fig. 3. The plasma level of keratan sulfate at 40 days of age was approximately $700 \mathrm{ng} \mathrm{mL}^{-1}$ but rapidly decreased to approximately $400 \mathrm{ng} \mathrm{mL}^{-1}$ at 70 days of age. It ranged from 200-300 ng mL ${ }^{-1}$ after 100 days of age. Furthermore, the plasma level of keratan sulfate at 40 days of age was significantly higher than at the other days of age. When comparing the results among the 3 male systems, the plasma levels of keratan sulfate for Strain C at 40 and 150 days of age were significantly higher than those for Strains A and B (Fig. 4). In the field of veterinary medicine, a study reported that there were no marked changes in the level of keratan sulfate in dogs with $O A$ and that its level was similar to those in the presence of other diseases such as disorders of the nervous system and tumors. This was possibly because dogs with early to late OA were analyzed (Fujiki et al., 2006). In horses with osteochondrosis, the level of keratan sulfate was markedly higher than in normal horses. However, there were no changes in its level in those with $O A$ (Todhunter et al., 1997).

The results of this experiment suggest that the pig serum level of keratan sulfate can be measured using a keratan sulfate ELISA kit for humans. The keratan sulfate level in juvenile pigs was higher and there was a decrease at an advanced age. In humans, the level of keratan sulfate rapidly increases from birth until 4 years of age and remains high at 12 years of age but gradually decreases from 15 until 20 years of age. Furthermore, there are no gender-disease type or race-related differences (Thonar et al., 1988). In horses, the level of keratan sulfate reaches a peak at 10 weeks of age when limb extension is the most marked but gradually decreases thereafter. A study indicated that there was a gender difference in horses and that the keratan sulfate level in males was significantly higher than in females (Okumura et al., 2002). In dogs, its level remains high from birth until 4 months of age but decreases until 9 months of age reaching a plateau (Fujiki et al., 2006). The results of measurement in pigs in this study are consistent with those of these studies with other animals, although the age at the peak differs. This suggests that cartilage formation is marked in juvenile pigs. It is known that fine ducts, cartilage ducts are present in young animals and that cartilage is provided with blood from blood vessels among these ducts (Ytrehus et al., 2004, 2007). Along with growth, cartilage is substituted for cancellous bone through ossification. The articular cartilage thickness gradually decreases and blood vessels distributed in cartilage gradually connect with each other, leading to the loss of vascular distribution (Ytrehus et al., 2007). As the serial changes in the plasma level of keratan sulfate in pigs resemble the kinetics of cartilage thickness earlier reported (Nakano et al., 1979) keratan sulfate provided from blood vessels distributed in the articular cartilage at young age to cartilage may have contributed to a high blood level of keratan sulfate.

The results of measurement in this study showed that the keratan sulfate level markedly decreased with parity differing from findings in other animals. A study reported that the mean level of keratan sulfate in healthy adults was $176.8 \pm 68.1 \mathrm{ng} \mathrm{mL}^{-1}$ (Hanashima et al., 1997). In this study, its level in female pigs that experienced delivery was relatively lower than in humans. In addition, the keratan sulfate level rapidly decreased to approximately $1 / 10$ of that during the breeding period. No study has reported such changes in its level in any animal.

This may have been associated with a pig-specific breeding form. After birth, pigs freely move around their mothers and are fed while conflicting with their brothers. 
After weaning, they also freely walk in a large pig house, eat food and drink water. However, after the start of breeding, the body weight and body-surface area increase with growth, gradually affecting movement. In addition, the breeding area becomes smaller than during the weaning period. In sows, movement is restricted with a farrowing crate and the stall. In particular, they do not stand up during lactation, restricting joint movement and reducing cartilage metabolism. In large animals, a large volume of articular synovial fluid can be collected. In addition, its production is greater in animals that freely move. Briefly, breeding under movement restriction may decrease the production of synovial fluid necessary for the synthesis and repair of the articular cartilage.

\section{CONCLUSION}

In pigs with leg weakness, the level of keratan sulfate was lower than in those with healthy limbs. This was consistent with the kinetics of the keratan sulfate level in humans with RA in a earlier study (Hanashima et al., 1997). This may reflect a reduction in the synthetic capacity for cartilage metabolism. Therefore, the plasma level of keratan sulfate may be reduced in pigs with leg weakness.

\section{REFERENCES}

Arai, S., S. Itoh, M. Kachi, Y. Sugiyama and S. Kusuhara, 2009. Clinical model of leg weakness induced by intraarticular administration of papain in pigs. Jpn. J. Swine Sci., 46: 183-189.

Arai, S., S. Itoh, T. Sugiyama, S. Kusuhara and F. Sunaga, 2010. Cartilage pathology of intra-articular papaininduced leg weakness in a pig model. Jpn. J. Swine Sci., 47: 136-138.

Fujiki, M., H. Kamiya, K. Arai, K. Misumi and H. Sakamoto, 2006. The effects of growth and disease in serum keratan sulfate concentration in dogs. J. Vet. Med. Sci., 68: 947-951.

Furugouri, H., 1984. Pigs leg weakness-cause diagnosis and prevention. J. Livest. Med., 256: 3-13.
Hanashima, M., K. Takagishi, Y. Gosou, K. Okamura and M. Itoman, 1997. Determination of serum and knee joint synovial fuluid keratan sulfate with a Sandwich-ELISA. Kitasato Med., 27: 246-256.

Kikuchi, T. and T. Hayakawa, 1992. The Potential of Cartilage Markers in Joint Fluid for Drug Evaluation. In: Articular Cartilage and Osteoarthritis, Kuettner, K.E., R. Schleyerbach, J.G. Peyron and V.C. Hascall (Eds.). Raven Press, New York, USA., pp: 597-609.

Kusuhara, S., 1999. Leg Weakness. 4th Edn., Kindai Shuppan, Tokyo Japanese, pp: 478-480.

Levick, J.R., 1990. The Clearance of Macromolecular Substances Such as Cartilage Markers from Synovial Fluid and Serum. In: Methods in Cartilage Research, Maroudas, A. and K. Kuettner (Eds.). Academic Press, London, UK., pp: 352-357.

Nakano, T., F.X. Aherne and J.R. Tompson, 1979. Changes in swine knee articular cartilage during growth. Can. J. Anim. Sci., 59: 167-179.

Okumura, M., M.G. Kim, H. Tagami, S. Haramaki and T. Fujinaga, 2002. Serum keratan sulphate as a cartilage metabolic in horses: The effect of exercise. J. Vet. Med. A. Physiol. Pathol. Clin. Med., 49: 195-197.

Reiland, S., 1978. Pathology of so-called leg weakness in the pig. Acta. Radiol. Suppl., 358: 23-44.

Thonar, E.J., L.M. Pachman, M.E. Lenz, J. Hayford, P. Lynch and K.E. Kuettner, 1988. Age related changes in the concentration of serum keratan sulphate in children. J. Clin. Chem. Clin. Biochem., 26: $57-63$.

Todhunter, R.J., S.L. Fubini, K.P. Freeman and G. Lust, 1997. Concentrations of keratan sulfate in plasma and synovial fluid from clinically normal horses and horses with joint disease. J. Am. Vet. Med. Assoc., 210: 369-374.

Ytrehus, B., C.S. Carlson and S. Ekman, 2007. Etiology and pathogenesis of osteochondrosis. Vet. Pathol., 44: 429-448.

Ytrehus, B., H.A. Haga, C.N. Mellum, L. Mathisen and C.S. Carlson et al., 2004. Experimental ischemia of porcine growth cartilage produces lesions of osteochondrosis. J. Orthopaedic Res., 22: 1201-1209. 